\title{
Rearrangements in the Genomes of Vibrio cholerae Strains Belonging to Different Serovars and Biovars
}

\author{
SUVOBROTO NANDI, GOPAL KHETAWAT, SANGHAMITRA SENGUPTA, RUMA MAJUMDER, \\ SUJATA KAR, RUPAK K. BHADRA, SUSANTA ROYCHOUDHURY, AND JYOTIRMOY DAS* \\ Biophysics Division, Indian Institute of Chemical Biology, Calcutta 700 032, India
}

\begin{abstract}
The intron-encoded enzyme I-CeuI provides an excellent tool for rapidly examining the organization of genomes of related species of bacteria. Vibrio cholerae strains belonging to serovars 01 and 0139 have 9 I-CeuI sites in their genomes, and $V$. cholerae strains belonging to serovars non-O1 and non-O139 have $10 \mathrm{I}-\mathrm{CeuI}$ sites in their genomes. This information can be used as a criterion to differentiate 01 strains from non-O1 and non-O139 strains. To our knowledge, intraspecies variation in the number of $r \boldsymbol{r n}$ operons has not been reported in any other organism. Our data revealed extensive restriction fragment length polymorphism based on a comparison of the I-CeuI digestion profiles of strains belonging to different serovars and biovars. From the analysis of partial digestion products, I-CeuI macrorestriction maps of several classical, El Tor, and 0139 strains were constructed. While the linkage maps are conserved within biovars, linkage maps vary substantially between biovars.
\end{abstract}

Vibrio cholerae, a noninvasive gram-negative bacterium, is the causative agent of the diarrheal disease cholera, which still is a public health problem in most developing countries. The specificity of the somatic $\mathrm{O}$ antigen of $V$. cholerae resides in the polysaccharide moiety of the lipopolysaccharide present in the outer membrane, which forms the basis for serological classification of this organism (16). Strains belonging to serovar $\mathrm{O}$ group 1 (serovar O1) are responsible for cholera. The strains of serovar $\mathrm{O} 1$ belong to two biovars, biovars classical and $\mathrm{El}$ Tor. Strains other than O1 strains are often called non-O1 vibrios, and these organisms can cause only sporadic infections and do not have the potential to cause epidemics (10). Only recently, an outbreak of cholera in the Indian subcontinent was caused by a novel non-O1 strain, $V$. cholerae O139 Bengal (1, 12). However, several lines of evidence have suggested that this strain very closely resembles biovar El Tor of serovar O1 $(2,3$, 18 ). By digesting the intact genomic DNA with enzymes NotI and $S f i \mathrm{I}$, which produced fragments of suitable sizes that could be resolved by pulsed-field gel electrophoresis (PFGE), the genome size of the classical $\mathrm{O} 1$ strains of $V$. cholerae was estimated to be about $3.2 \mathrm{Mb}$ (14). A physical map of the genome of strain $569 \mathrm{~B}$ was constructed by using restriction endonucleases NotI, SfiI, and I-CeuI, and on this map 20 cloned homologous and heterologous genes and seven $\mathrm{mm}$ operons were positioned (8).

I-CeuI is encoded by a class I mobile intron (9). I-CeuI digestion followed by PFGE can be a useful tool for examining intra- and interspecies genome rearrangements (5). This enzyme cleaves genomic DNA only in a 19-bp sequence in the $23 \mathrm{~S}$ rRNA gene of the $r m$ operon in all of the organisms examined so far (6). The number of I-CeuI sites in the genome of an organism is considered a measure of the number of $\mathrm{rm}$ operons present in that organism. The number and location of $m n$ operons in enteric bacteria appear to be highly conserved. The I-CeuI restriction maps reported for a number of Escherichia coli and Salmonella typhimurium strains are almost identical $(5,6)$. However, the I-CeuI maps of Salmonella typhi Ty2

${ }^{*}$ Corresponding author. Mailing address: Biophysics Division, Indian Institute of Chemical Biology, 4, Raja S. C. Mullick Road, Calcutta 700 032, India. Phone: (91-33) 473 0350. Fax: (91-33) 4730350. E-mail:iicb\%sirnetc@sirnetd.ernet.in. and $S$. typhimurium LT2 revealed chromosomal rearrangements in these two species (7). In the present report we describe strains of $V$. cholerae belonging to serovars $\mathrm{O} 1$ and $\mathrm{O} 139$ that have $9 \mathrm{I}-\mathrm{Ce} u \mathrm{I}$ sites in their genomes and $V$. cholerae strains belonging to serovar non-O1 that have $10 \mathrm{I}-\mathrm{CeuI}$ sites in their genomes. Although the number of $r m$ operons in strains belonging to serovar $\mathrm{O} 1$ is conserved, the positions of the operons in the chromosome are different, suggesting that there have been major genomic rearrangements in the classical and El Tor biovars.

\section{MATERIALS AND METHODS}

Bacterial strains and growth conditions. The $V$. cholerae strains used in this study are listed in Table $1 . V$. cholerae cells were grown in a gyratory shaker at $37^{\circ} \mathrm{C}$ in nutrient broth containing $0.1 \mathrm{M} \mathrm{NaCl}(\mathrm{pH} \mathrm{8.0)}$ and were maintained as described previously (13). E. coli cells were grown in Luria-Bertani medium with shaking at $37^{\circ} \mathrm{C}$.

Preparation of high-molecular-weight genomic DNA and enzyme digestion. Agarose plugs of cells in the logarithmic phase of growth were prepared by mixing equal volumes of cell suspension and molten $1.4 \%$ low-melting-point agarose as described previously $(8,14)$. The plugs were suspended in lysis solution (8) and incubated at $37^{\circ} \mathrm{C}$ for $16 \mathrm{~h}$ with gentle shaking. After the incubation period, the lysis solution was replaced by a solution containing $0.5 \mathrm{M}$ EDTA ( $\mathrm{pH}$ 9.0), $1 \%$ Sarkosyl, and $1 \mathrm{mg}$ of proteinase $\mathrm{K}$ per $\mathrm{ml}$, and the preparation was incubated for $48 \mathrm{~h}$ at $50^{\circ} \mathrm{C}$, following which the plugs were stored in the same solution at $4^{\circ} \mathrm{C}$. The agarose plugs containing the genomic DNA were digested with I-CeuI as described previously (8). For partial digestion with I-CeuI, six agarose pieces were digested with $1 \mathrm{U}$ of the enzyme in $100 \mu \mathrm{l}$ of the reaction mixture for $2 \mathrm{~h}$ at $37^{\circ} \mathrm{C}$.

PFGE and hybridization experiments. Electrophoresis was carried out with a Pulsaphor Plus System with a hexagonal electrode array (Pharmacia, Uppsala, Sweden) as described previously $(8,14)$. FastLane agarose (FMC Corp., Philadelphia, Pa.) was used when DNA was to be transferred to membranes for Southern blotting. The enzyme-digested DNA was separated on a $1 \%$ agarose gel by using $20 \mathrm{mM}$ Tris-acetate ( $\mathrm{pH} 8.3$ )- $0.5 \mathrm{mM}$ EDTA (TAE) as the running buffer at 5 to $10 \mathrm{~V} / \mathrm{cm}$ (depending on the size of the fragment that needed to be resolved) and various pulse times (depending on the experimental conditions). Phage $\lambda$ multimeric DNA and yeast chromosomal DNA were used as molecular mass markers. Preparation of ${ }^{32} \mathrm{P}$-labeled DNA probes, $r p o H$ (encoding $\sigma^{32}$ of $V$. cholerae [15]), mutL, and NotI-linking clone NLH20 of V. cholerae genomic DNA (8) and Southern blot hybridizations were carried out by using previously described procedures (8). Details concerning the cloned DNA probes used in this study have been described previously (8). End labeling of DNA fragments following I-CeuI digestion was done by incubating the agarose blocks in a buffer containing Klenow enzyme and $\left[\alpha^{32} \mathrm{P}\right] \mathrm{dCTP}$, and the preparations were subjected to PFGE followed by autoradiography. 
TABLE 1. V. cholerae strains used

\begin{tabular}{|c|c|c|c|c|c|c|c|}
\hline \multirow{2}{*}{ Strain designation as received } & \multirow{2}{*}{ Serovar } & \multirow{2}{*}{ Biovar } & \multirow{2}{*}{$\begin{array}{c}\text { CT } \\
\text { gene }^{a}\end{array}$} & \multicolumn{3}{|c|}{ Origin } & \multirow{2}{*}{ Sender } \\
\hline & & & & Source & Place & Year & \\
\hline 569B (= ATCC 25870) & O1 & Classical & $\mathrm{CT}^{+}$ & Clinical & India & 1948 & NICED, Calcutta, India ${ }^{b}$ \\
\hline O395 (= ATCC 39541) & O1 & Classical & $\mathrm{CT}^{+}$ & Clinical & India & 1964 & J. J. Mekalanos, United States \\
\hline $\mathrm{VC} 44$ & $\mathrm{O} 1$ & El Tor & $\mathrm{CT}^{+}$ & Clinical & India & 1992 & G. B. Nair, India \\
\hline $\mathrm{CO} 457$ & O1 & El Tor & $\mathrm{CT}^{+}$ & Clinical & India & 1994 & G. B. Nair, India \\
\hline C6709 & O1 & El Tor & $\mathrm{CT}^{+}$ & Clinical & Peru & 1991 & J. J. Mekalanos, United States \\
\hline MAK757 (= ATCC 51352) & $\mathrm{O} 1$ & El Tor & $\mathrm{CT}^{+}$ & Clinical & Indonesia & 1961 & NICED, Calcutta, India \\
\hline MO45 (= ATCC 51394) & O139 & & $\mathrm{CT}^{+}$ & Clinical & India & 1992 & G. B. Nair, India \\
\hline SG24 & O139 & & $\mathrm{CT}^{+}$ & Clinical & India & 1992 & G. B. Nair, India \\
\hline V18 & O11 & & $\mathrm{CT}^{-}$ & Clinical & India & 1989 & G. B. Nair, India \\
\hline RB & Non-O1, non-O139 & & $\mathrm{CT}^{-}$ & Clinical & India & 1991 & G. B. Nair, India \\
\hline V254 & Non-O1, non-O139 & & $\mathrm{CT}^{-}$ & Clinical & India & 1991 & G. B. Nair, India \\
\hline NRT36 & Non-O1, non-O139 & & $\mathrm{CT}^{-}$ & Clinical & Japan & 1986 & G. B. Nair, India \\
\hline VCE232 & Non-O1, non-O139 & & $\mathrm{CT}^{+}$ & Environmental & India & 1985 & G. B. Nair, India \\
\hline VCE309 & Non-O1, non-O139 & & $\mathrm{CT}^{-}$ & Environmental & India & 1985 & G. B. Nair, India \\
\hline
\end{tabular}

${ }^{a} \mathrm{CT}$, Cholera toxin gene.

${ }^{b}$ NICED, National Institute of Cholera and Enteric Diseases.

\section{RESULTS AND DISCUSSION}

I-CeuI map of strain 569B. It has been reported that I-CeuI digestion of $V$. cholerae 569B and 0395 produced seven fragments ranging in size from 1,420 to $70 \mathrm{~kb}(8)$. In the course of our studies on the construction of a macrorestriction map of the $V$. cholerae genome and for better resolution of DNA fragments following enzyme digestion, refinement of PFGE methods by altering various parameters, such as the nature of pulsing, pulse time, and time of electrophoresis, was necessary. One of the modified procedures revealed that previously described I-CeuI fragment C7 is a doublet comprising 85- and $80-\mathrm{kb}$ fragments. After $V$. cholerae 569B genomic DNA in an agarose block was digested with I-CeuI and end labeled with $\left[\alpha-{ }^{32} \mathrm{P}\right] \mathrm{dCTP}$, after the fragments were separated by PFGE,

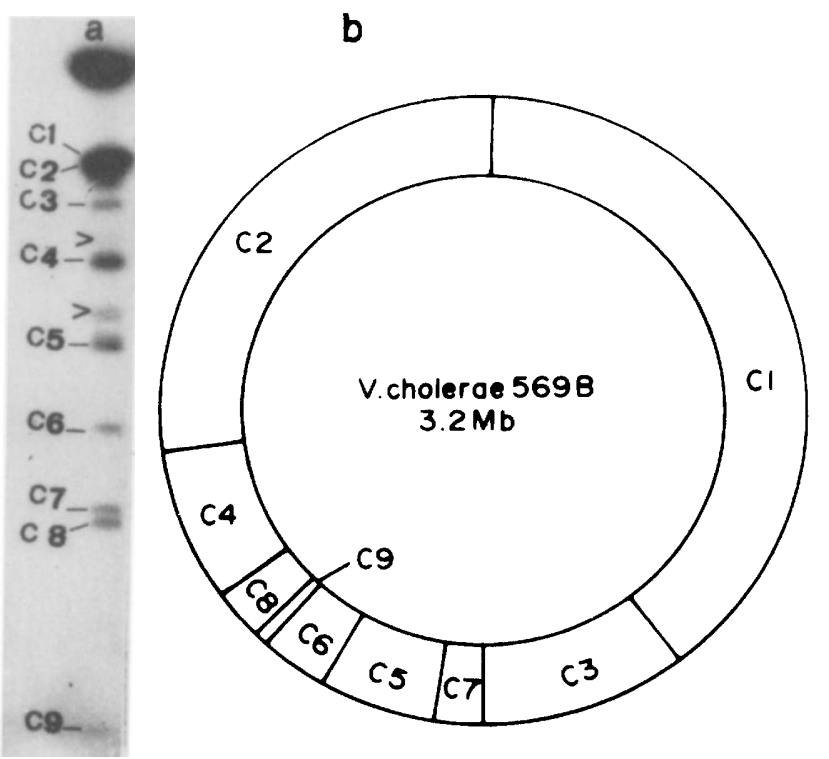

FIG. 1. (a) Autoradiogram of PFGE-separated $V$. cholerae 569B genome digested with I-CeuI either partially or to completion. Digested DNAs in agarose blocks were end labeled with $\left[\alpha{ }^{-32} \mathrm{P}\right] \mathrm{dCTP}$ and separated in $1 \%$ FastLane agarose (FMC) containing $0.5 \times$ TAE, with the pulse times ramping between 5 and $25 \mathrm{~s}$ for $22 \mathrm{~h}$ at $10 \mathrm{~V} / \mathrm{cm}$. Fragment numbers are indicated on the left. The arrowheads indicate the partial digestion products. (b) I-CeuI map of $V$. cholerae 569B genome. and after the dried gel was autoradiographed, we confirmed that I-CeuI fragment $\mathrm{C} 7$ is a doublet and detected another small, 6-kb fragment which was not visible in the ethidium bromide-stained gels (Fig. 1a). Under identical conditions, I$C e u I$ digestion of the $E$. coli W3110 genome produced only seven fragments, as reported previously (5). Thus, the $V$. cholerae 569B genome has nine I-CeuI sites instead of the seven sites reported previously (8). To examine whether, as in other organisms, the I-CeuI sites in the $V$. cholerae genome are also located only in the $m$ operons, a 450 -bp region of the $23 \mathrm{~S}$ rRNA gene of $V$. cholerae (characterization of this gene will be described in a separate report) was used as a probe for Southern blot hybridization of the I-CeuI-digested $V$. cholerae $569 \mathrm{~B}$ genome. All nine fragments hybridized with the probe (data not shown), confirming that there are nine $r r n$ operons in the $V$. cholerae 569B genome and that I-CeuI does not have sites that are not in the rrn operon. The other classical strain examined in this study, O395, also has nine I-CeuI sites in its genome.

To position the two additional fragments on the I-CeuI macrorestriction map of the $V$. cholerae 569B genome (8), a restriction map was reconstructed from the results of the analysis of partial digestion products. Eleven partial fragments (Table 2) obtained by using different pulse times during PFGE were analyzed to assemble the I-CeuI map. From partial fragments P10 and P11 the linkage C2-C1-C3 was established. These partial fragments, along with partial fragments $\mathrm{P} 5$ and $\mathrm{P} 7$, gave the linkage C2-C1-C3-C7-C5. When partial fragments $\mathrm{P} 1, \mathrm{P} 2$, $\mathrm{P} 3$, and P4 were combined, the linkages between I-CeuI frag-

TABLE 2. I-CeuI partial digestion products of the 569B genome

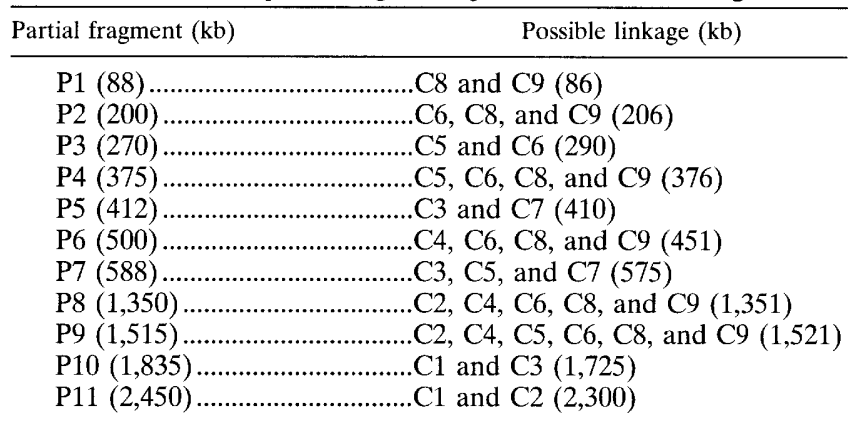




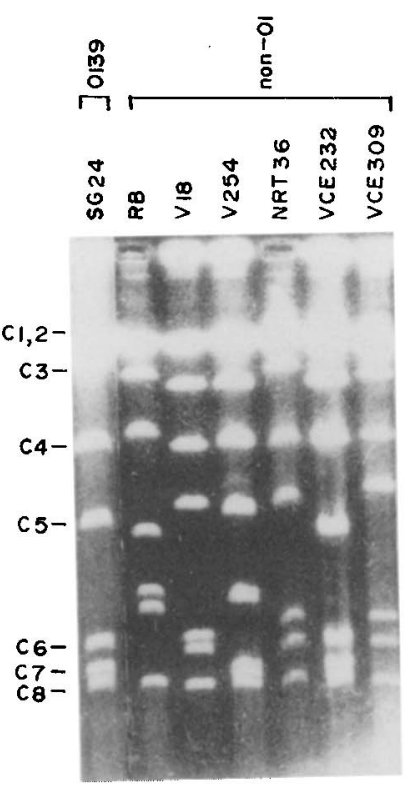

$A$

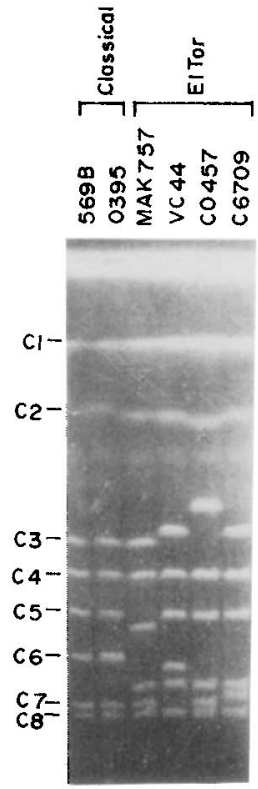

B

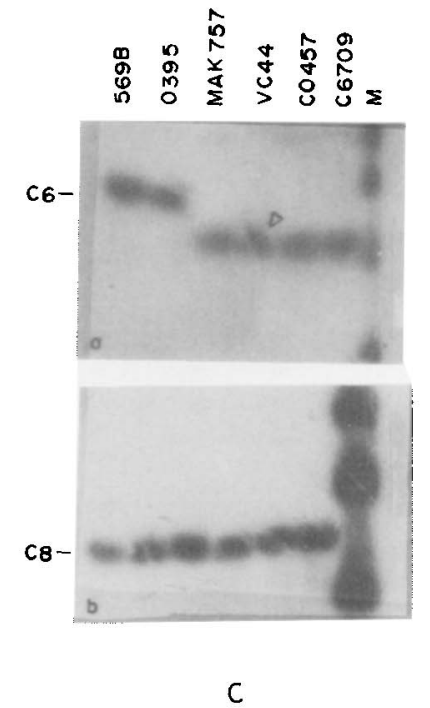

FIG. 2. (A and B) I-CeuI digestion profiles of the genomes of $V$. cholerae strains belonging to different serovars and biovars. Enzyme-digested DNAs were separated by using $1 \%$ FastLane agarose (FMC) and $0.5 \times \mathrm{TAE}$, with pulse times ramping from 5 to $25 \mathrm{~s}$ for $22 \mathrm{~h}$ at $10 \mathrm{~V} / \mathrm{cm}$ (A) or with pulse times of $5 \mathrm{~s}$ (for $4 \mathrm{~h}$ ), $10 \mathrm{~s}$ (for $4 \mathrm{~h}$ ), $20 \mathrm{~s}$ (for $4 \mathrm{~h}$ ), and $100 \mathrm{~s}$ (for $12 \mathrm{~h}$ ) (B). (C) Southern blot hybridization of the gel in panel B by using rpoH (arrowhead indicates fragment C7 of the I-CeuI-digested VC44 genome) (a) and NLH20 (b) as the probes. The fragment numbers are indicated on the left. Lane M contained $\lambda$ concatameric DNA used as a marker.

ments were found to be $\mathrm{C} 2-\mathrm{C} 1-\mathrm{C} 3-\mathrm{C} 7-\mathrm{C} 5-\mathrm{C} 6-\mathrm{C} 9-\mathrm{C} 8$. Finally, by using partial fragments $\mathrm{P} 6, \mathrm{P} 8$, and $\mathrm{P} 9$, the I-CeuI linkage map of strain 569B was constructed (Fig. 1b). The linkages were confirmed by using I-CeuI fragment-specific DNA probes. For example, when a C6-specific probe, mutL (8), was hybridized to a Southern blot of the $V$. cholerae 569B genome partially digested with I-CeuI, it hybridized with partial fragments $\mathrm{P} 2, \mathrm{P} 3, \mathrm{P} 4, \mathrm{P} 6, \mathrm{P} 8$, and $\mathrm{P} 9$. The $r p o H$ gene of $V$. cholerae 569B (15), which is also a C6-specific probe, hybridized with partial fragments P2, P3, and P4. Similarly, when the C8specific probe, linking clone NLH 20 (8), which links N11 and N25 fragments of the NotI-digested 569B genome (8), was used as a probe in the hybridization experiment, it hybridized with partial fragments $\mathrm{P} 2, \mathrm{P} 4, \mathrm{P} 6, \mathrm{P} 8$, and $\mathrm{P}$. These results show that $\mathrm{C} 6, \mathrm{C} 9$, and $\mathrm{C} 8$ are linked. The I-CeuI linkage maps of strains 569B and O395 were identical.

Comparison of the I-CeuI digestion profiles of genomes of $V$. cholerae strains. Since I-CeuI can serve as a tool to examine the genome organization of related strains, the I-CeuI digestion profiles of $V$. cholerae cells belonging to different serovars and biovars were compared (Fig. 2A and B). While the genomes of classical and El Tor strains have 9 I-CeuI sites, all non-O1 and non-O139 strains examined have $10 \mathrm{I}-\mathrm{Ceu} \mathrm{I}$ sites in their genomes (Table 3 and Fig. 3). The difference in the number of $\mathrm{I}-\mathrm{Ce} \mathrm{I}$ sites in the genomes of strains belonging to the $\mathrm{O} 1$ serovar and the non-O1 and non-O139 serovars can thus be used as a criterion to differentiate them. Interestingly, strain SG24 belonging to serogroup $\mathrm{O} 139$ has $9 \mathrm{I}-\mathrm{CeuI}$ sites in its genome, while the other non-O1 strains have 10 sites. The I-CeuI digestion profile of the SG24 genome resembles that of O1 El Tor strain CO457 (Fig. 3, lanes a and g), a strain isolated immediately after the $\mathrm{O} 139$ epidemic. To our knowledge, intraspecies variation in the number of $r r n$ operons has not been reported in any other organism. Some laboratory-maintained strains of Bacillus subtilis, a gram-positive bacterium, have been reported to have $9 \mathrm{rm}$ operons rather than the more

TABLE 3. I-CeuI fragment sizes for the genomes of different $V$. cholerae strains

\begin{tabular}{|c|c|c|c|c|c|c|c|c|c|c|}
\hline \multirow{2}{*}{ Strain } & \multicolumn{10}{|c|}{ Fragment sizes $(\mathrm{kb})$} \\
\hline & $\mathrm{C} 1$ & $\mathrm{C} 2$ & C3 & $\mathrm{C} 4$ & $\mathrm{C} 5$ & C6 & $\mathrm{C} 7$ & $\mathrm{C} 8$ & $\mathrm{C} 9$ & $\mathrm{C} 10$ \\
\hline $569 \mathrm{~B}$ & 1,400 & 900 & 325 & 245 & 170 & 120 & 85 & 80 & 6 & \\
\hline O395 & 1,400 & 900 & 325 & 245 & 170 & 120 & 85 & 80 & 6 & \\
\hline MAK757 & 1,400 & 790 & 325 & 245 & 150 & 92 & 85 & 80 & 6 & \\
\hline VC44 & 1,400 & 790 & 364 & 245 & 170 & 92 & 90 & 80 & 6 & \\
\hline $\mathrm{CO} 457$ & 1,400 & 750 & 460 & 245 & 170 & 92 & 84 & 80 & 6 & \\
\hline C6709 & 1,400 & 820 & 364 & 245 & 170 & 94 & 88 & 80 & 6 & \\
\hline SG24 & 1,400 & 750 & 460 & 245 & 170 & 92 & 84 & 80 & 6 & \\
\hline $\mathrm{RB}$ & 1,400 & 950 & 350 & 254 & 164 & 116 & 106 & 80 & 6 & 5 \\
\hline V18 & 1,400 & 820 & 340 & 245 & 192 & 98 & 92 & 80 & 6 & 5 \\
\hline V254 & 1,400 & 820 & 340 & 245 & 180 & 116 & 84 & 80 & 6 & 5 \\
\hline NRT36 & 1,400 & 750 & 364 & 245 & 196 & 106 & 92 & 80 & 6 & 5 \\
\hline VCE232 & 1,400 & 950 & 340 & 245 & 168 & 94 & 84 & 80 & 6 & 5 \\
\hline
\end{tabular}




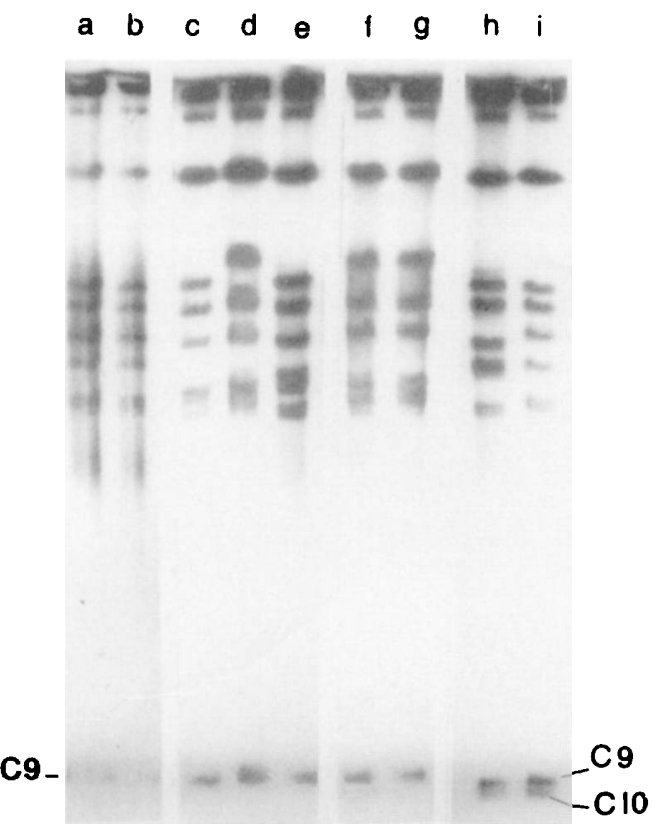

FIG. 3. Autoradiograph of I-CeuI-digested genomes of $V$. cholerae $\mathrm{O} 1 \mathrm{El}$ Tor, non-O1 and non-O139 strains and O139 strains following end labeling with $\left[\alpha{ }^{32} \mathrm{P}\right] \mathrm{dCTP}$ of the digestion products and PFGE. Lane a, 569B; lane b, O395; lane c, C6709; lane d, CO457; lane e, VC44; lane f, MO45; lane g, SG24; lane h, RB; lane i, V254. Electrophoresis was carried out by using pulse times of $5 \mathrm{~s}$ (for $4 \mathrm{~h}$ ), $10 \mathrm{~s}$ (for $4 \mathrm{~h}$ ), $25 \mathrm{~s}$ (for $4 \mathrm{~h}$ ), and $100 \mathrm{~s}$ (for $11 \mathrm{~h}$ ) at $10 \mathrm{~V} / \mathrm{cm}$, followed by pulse times of $10 \mathrm{~s}$ (for $7 \mathrm{~h}$ ) and $150 \mathrm{~s}$ (for $6 \mathrm{~h}$ ) at $5 \mathrm{~V} / \mathrm{cm}$ at $3^{\circ} \mathrm{C}$. C9 and $\mathrm{C} 10$ indicate the positions of I-CeuI fragments 9 and 10 , respectively.

common $10 \mathrm{rrn}$ operons $(4,17)$. It was proposed that homologous recombination between closely linked rRNA genes caused deletion of one of the loci (19). The number of $r m$ operons in the other organisms examined so far is seven or less (4).

No detectable restriction fragment length polymorphism (RFLP) was observed in the two $\mathrm{O} 1$ classical strains examined (Fig. 2B). The four El Tor strains, on the other hand, exhibited a high degree of RFLP. Strains VC44 and CO457 exhibited extensive RFLP, which is surprising in view of the fact that these two strains were isolated within a span of 1.5 years immediately before and after the O139 epidemic (11). Strain C6709, which was isolated during the Peru epidemic in 1991, is different from other El Tor strains. The I-CeuI digestion profiles of the non-O1 and non-O139 strains examined showed extensive RFLP, which were more pronounced among the low-molecular-weight fragments (Fig. 2A).

When I-CeuI fragment-specific probes were used in Southern blot hybridization experiments with I-CeuI-digested genomic DNAs from a number of O1 classical and El Tor strains, the extent of RFLP could be detected in greater detail. When the rpoH gene of $V$. cholerae 569B (15), which hybridizes with the $\mathrm{C} 6$ fragment of the $569 \mathrm{~B}$ genome, was used as a probe, it hybridized with the $120-\mathrm{kb}$ C6 fragment of 569B and $\mathrm{O} 395$ and the 92-kb C6 fragment of all of the El Tor strains except VC44 (Table 3 ). Thus, there is an $\sim 30-\mathrm{kb}$ deletion in the C6 fragment of the $\mathrm{O} 1 \mathrm{El}$ Tor strains compared to the C6 fragment of $\mathrm{O} 1$ classical strains. The deletion or insertion of DNA fragments presumably occurred via mobile elements and resulted in the observed RFLP differences between strains. In the genomic digest of $\mathrm{VC} 44$, the $r p o H$ probe hybridized with 92-kb fragment C7 (Fig. 2Ca). Fragment C7 of VC44 comigrated with fragment $\mathrm{C} 6$ of other $\mathrm{El}$ Tor strains (Fig. 2B).

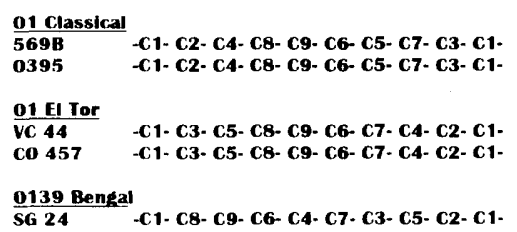

FIG. 4. I-CeuI linkage map of genomes of $V$. cholerae 569B, O395, VC44, $\mathrm{CO} 457$, and SG24.

Linking clone NLH 20 (8), a C8-specific probe, hybridized to 80 -kb fragment $\mathrm{C} 8$ of all classical and $\mathrm{El}$ Tor strains (Fig. 2Cb). The secY gene of $V$. cholerae, located in fragment $C 4(8)$, hybridized to $245-\mathrm{kb}$ fragment $\mathrm{C} 4$ of all of the strains belonging to the classical and El Tor biovars.

The I-CeuI macrorestriction maps of several classical, El Tor, and O139 strains were constructed by analyzing the I-CeuI partial digestion products (Fig. 4). The linkage of the I-CeuI fragments of the genomes of all of the classical strains and the linkage of all of the El Tor strains examined are conserved. However, the linkages are different between the strains belonging to the two biovars. These results suggest that although the number of $r m$ operons is conserved among strains belonging to serovar $\mathrm{O} 1$, the positions of the operons in the chromosome have changed, suggesting that there have been major genomic rearrangements in the classical and $\mathrm{El}$ Tor biovars. The I-CeuI linkage map of the genome of strain SG24 is different from the I-CeuI linkage maps of the genomes of the classical and El Tor strains (Fig. 4), although the I-CeuI digestion profile of strain SG24 is similar to that of El Tor strain CO457.

\section{ACKNOWLEDGMENTS}

We thank G. B. Nair, National Institute of Cholera and Enteric Diseases, Calcutta, India, for providing the $V$. cholerae strains and $\mathrm{J} . \mathrm{J}$ Mekalanos, Harvard Medical School, Boston, Mass., for strain C6709. We are grateful to all members of the Biophysics Division, Indian Institute of Chemical Biology, for their kind cooperation and encouragement during this study, particularly to Rajat Bancrjee and Amit Chakraborty.

This work was supported by grants BT/TF/15/03/91 and BT/MB/05/ 012/94 from the Department of Biotechnology, Government of India. S.N. and R.M. are grateful to the Council of Scientific \& Industrial Research, New Delhi, India, for predoctoral fellowships, and S.K. is grateful for a postdoctoral fellowship.

\section{REFERENCES}

1. Albert, M. J., A. K. Siddique, M. S. Islam, A. S. J. Faruque, M. Ansurazzaman, S. M. Faruque, and R. B. Sack. 1993. Large outbreak of clinical cholera due to Vibrio cholerae non-O1 in Bangladesh. Lancet 341:704.

2. Bhadra, R. K., S. Roychoudhury, R. K. Banerjee, S. Kar, R. Majumdar, S. Sengupta, S. Chatterjee, G. Khetawat, and J. Das. 1995. Cholera toxin (CTX) genetic element in Vibrio cholerae O139. Microbiology 141:19771983.

3. Bhadra, R. K., S. Roychoudhury, and J. Das. 1994. Vibrio cholerae O139 El Tor biotype. Lancet 343:728.

4. Krawiec, S., and M. Riley. 1990. Organization of the bacterial genome. Microbiol. Rev. 54:502-539.

5. Liu, S.-L., A. Hessel, and K. E. Sanderson. 1993. Genomic mapping with $\mathrm{I}-\mathrm{CeuI}$, an intron-encoded endonuclease specific for genes for ribosomal RNA, in Salmonella spp., Escherichia coli and other bacteria. Proc. Natl. Acad. Sci. USA 90:6874-6878.

6. Liu, S.-L., and K. E. Sanderson. 1995. I-CeuI reveals conservation of the genome of independent strains of Salmonella typhimurium. J. Bacteriol. 177:3355-3357.

7. Liu, S.-L., and K. E. Sanderson. 1995. Rearrangements in the genome of the bacterium Salmonella typhi. Proc. Natl. Acad. Sci. USA 92:1018-1022.

8. Majumder, R., S. Sengupta, G. Khetawat, R. K. Bhadra, S. Roychoudhury, and J. Das. 1996. Physical map of the genome of Vibrio cholerae 569B and localization of genetic markers. J. Bacteriol. 178:1105-1112.

9. Marshall, P., and C. Lemieux. 1991. Cleavage pattern of the homing endo- 
nuclease encoded by the fifth intron in the chloroplast large subunit rRNAencoding gene of Chlamydomonas eugametos. Gene 104:241-245.

10. Morris, J. G. 1990 . Non-O group I Vibrio cholerae: a look at the epidemiology of an occasional pathogen. Epidemiol. Rev. 12:179-191.

11. Nair, G. B. Personal communication.

12. Ramamurthy, T., S. Garg, R. Sharma, S. K. Bhattacharya, G. B. Nair, T. Shimada, T. Takeda, T. Karasawa, H. Kurazano, A. Pal, and Y. Takeda. 1993. Emergence of novel strain of Vibrio cholerae with epidemic potential in southern and eastern India. Lancet 341:703-704.

13. Roy, N. K., G. Das, T. S. Balganesh, S. N. Dey, R. K. Ghosh, and J. Das. 1982 Enterotoxin, DNA repair and alkaline phosphatase of Vibrio cholerae before and after animal passage. J. Gen. Microbiol. 128:1927-1932.

14. Roychoudhury, S., R. K. Bhadra, and J. Das. 1994. Genome size and restriction fragment length polymorphism analysis of Vibrio cholerae strains belonging to different serovars and biotypes. FEMS Microbiol. Lett. 115: 329-334.

15. Sahu, G. K., R. Chowdhury, and J. Das. The $r p o H$ gene encoding $\sigma^{32}$ homolog of Vibrio cholerae. Gene, in press.

16. Shimada, T., E. Arakawa, K. Itoh, T. Okitsu, A. Matsushima, Y. Asai, S. Yamai, T. Nakazato, G. B. Nair, M. J. Albert, and Y. Takeda. 1994. Extended serotyping scheme for Vibrio cholerae. Curr. Microbiol. 28:175-178.

17. Toda, T., and M. Itaya. 1995. I- $\mathrm{CeuI}$ recognition sites in the $r m$ operons of the Bacillus subtilis 168 chromosome: inherent landmarks for genome analysis. Microbiology 141:1937-1945.

18. Waldor, M., and J. J. Mekalanos. 1994. ToxR regulates virulence gene expression in non-O1 strains of Vibrio cholerae that cause epidemic cholera. Infect. Immun. 62:72-78.

19. Widom, R. L., E. D. Jarvis, G. LaFauci, and R. Rudner. 1988. Instability of rRNA operons in Bacillus subtilis. J. Bacteriol. 170:605-610. 